\title{
The Effectiveness of a Program of Physical Activity and Diet to Modify Cardiovascular Risk Factors in Patients with Severe Mental Illness (CAPiCOR Study)
}

Roser Masa Font ${ }^{1}$, Maria Isabel Fernández Sanmartín², Luis Miguel Martín López ${ }^{3}$, Noemí Olona Tabueña ${ }^{4}$, Sílvia Oller Canet ${ }^{5}$, Luisa San Emeterio Echevarría ${ }^{6}$, Montserrat Ibarra Jato7, Belén Díaz Múgjica8, Susana González Tejón ${ }^{9}$, Lidia Viñas Cabrera ${ }^{10}$, Celia Tajada Vitales ${ }^{11}$, Antonio Manuel Alba Muñoz ${ }^{12}$, Rosa Sanchís Catalán ${ }^{8}$, Albert Barroso García ${ }^{13}$, Jaume Martín Royo ${ }^{14}$, Teresa Salvador Barbarroja ${ }^{6}$

\section{Abstract}

Background: Patients with severe mental disorders have a higher prevalence of cardiovascular risk factors. Obesity and sedentarism are cardiovascular risk factors and their control reduces morbidity and mortality. Thus, interventions directed toward decreasing weight and/ or increasing the level of physical activity are necessary.

Objectives: The aim of this study would be to evaluate the effectiveness of an educational intervention focused on diet and physical activity in order to change the amount of physical activity, Body Mass Index and waist circumference in these patients.

Design: Randomized clinical trial with a control group with one-year follow-up.

Setting: Outpatient Mental Health Teams of Barcelona and a residence for patients with severe mental disorders.

Participants: Patients between 18 and 65 years of age diagnosed with schizophrenia, a schizoaffective disorder or bipolar disorder in treatment with antipsychotic medication and a low level of physical activity (240 patients in each randomized group).
1 EAP Besòs, Institut Català de la Salut (ICS), Barcelona, Spain; Universitat Autònoma de Barcelona, Bellaterra, Spain.

2 Unitat Docent de Medicina Familiar i Comunitària, Barcelona, Spain.

3 Centre Institut de Neuropsiquiatria i Addiccions (INAD). Barcelona, Spain.

4 Àmbit d'Atenció Primària Barcelona Ciutat. Unitat d'Avaluació, Sistemes d'Informació i Qualitat, Barcelona, Spain.

5 Centre Salut Mental Maragall (INAD), Barcelona, Spain.

6 Centre Salut Mental Sant Martí Nord (INAD), Barcelona, Spain.

7 Centre Salut Mental Martí i Julià (INAD), Santa Coloma de Gramenet, Spain.

8 Centre Salut Mental Sant Martí Sud (INAD), Barcelona, Spain.

9 EAP Raval Sud (ICS), Barcelona, Spain 10 EAP Poblenou (ICS), Barcelona, Spain.

11 EAP Doctor Vilaseca (Can Mariner), Santa Coloma de Gramenet, Spain.

12 Centre Salut Mental Ciutat Vella, Parc Sanitari Sant Joan de Déu, Barcelona, Spain.

13 EAP Sant Martí (ICS), Barcelona, Spain.

14 CUAP Casernes (ICS), Barcelona, Spain.

Contact information:

\section{Roser Masa Font.}

Address: EAP Besòs, Institut Català de la Salut (ICS), C/Alfons el Magnànim, 57, 08019, Barcelona, Spain.

Tel: (34) 605579099

झ rosermasa@gmail.com 
Intervention group: Physical activity group educational program of 24 sessions over 12 weeks and diet (16 sessions in the first 8 weeks) carried out by nurses and physical activity specialists.

Control group: Usual practice with regular checks and the usual treatment of their disease.

Main outcome measures: Level of physical activity (IPAQ questionnaire), weight, Body Mass Index and waist circumference.

Other outcomes: Cardiovascular risk, quality of life (SF-36 questionnaire), tobacco consumption, dietary habit (PREDIMED questionnaire), blood pressure and laboratory parameters (cholesterol, triglycerides, glucose).

Evaluations will be masked and will be made at 0, 3, 6 and 12 months.

Data analysis: Intention to treat analysis. Analysis of variance for repeated measures to adjust for differences attributable to the effect of the intervention for potential confounders (drug treatment, care level of intervention and mental status of the patient).

Ethical aspects: The project has been evaluated and approved by the ethics committee (CEIC) of the Primary Healthcare-University Research Institute IDIAP Jordi Gol, with registration number P11/64.

Trial registration: Clinicaltrials.gov NCT01729650.

\author{
Keywords \\ Bipolar disorder, \\ cardiovascular risk, diet, \\ metabolic syndrome, obesity, \\ physical activity, quality of life, \\ schizophrenia, \\ severe mental disorder, \\ waist circumference.
}

\section{Background}

Schizophrenia, schizoaffective and bipolar disorders included in the group of severe mental disorders [SMD] have an overall prevalence of $3 \%$ in the general population [1].

Despite being a predominantly young population, in comparison to the general population, these patients have a significantly higher presence of cardiovascular risk factors [CVRF] such as smoking, obesity, dyslipidemia, diabetes mellitus or metabolic syndrome [MS] [2,3] along with a decrease in life expectancy of between 10 and 25 years [4].
This increased risk is due to the interaction of multiple etiological factors [5]: Genetic predisposition [6], environmental factors due to unhealthy lifestyles (bad dietary habits, smoking and sedentarism) [7] and the increasingly evident contribution of antipsychotic drugs in metabolism disorders, especially clozapine and olanzapine [8].

Obesity and sedentarism are independent CVRF. Their role in terms of cardiovascular risks has been well documented (Framingham Heart Study) [9]. However, they are also associated with many other diseases (osteoarthritis, cholelithia- 
sis, cancer, hepatic steatosis, peripheral vascular disease, psychological disorders and decreased quality of life, etc.). The most accepted method to define and classify obesity is the Body Mass Index [BMI] [10]. However, determining the waist circumference [WC] or abdomen circumference is also important as the risks associated with obesity not only depend on total adiposity, but its distribution (android), other CVRF and other comorbidities as well [5]. Obesity and overweight are two to three times more prevalent in patients with psychotic disorders. It is mostly due to abdominal type obesity [3, 11].

Interventions that have proven effective have been carried out to decrease the cardiovascular risk of patients with psychosis. A systematic review of 2010 that collected 21 randomized clinical trials of interventions to reduce or give up smoking in schizophrenia patients concluded that bupropion is able to bring about a reduction in the consumption of tobacco when compared to a placebo [12].

Other reviews of interventions aimed at reducing body weight or increasing the level of physical activity [PA] are less conclusive because of the small number of studies included, the small sample size or the heterogeneity of the variables collected. In a 2007 review by Cochrane [13], three randomized clinical trials were included but they had small sample sizes and short follow-ups. In another review that included 15 clinical trials, the outcome variables are highly heterogeneous [14]. Then again, in a review from 2012 with studies using similar response variables, sample sizes are very small [15]. Nevertheless, all these reviews suggested a benefit from these interventions.

Wu evaluated the effect of diet monitoring and exercise in obese patients with schizophrenia treated with olanzapine $(n=53)$ in which an improvement in metabolic profile and significant decrease in weight, BMI and WC was seen [16]. Daumit recently conducted an intervention in almost 300 patients with a severe mental disorder which revealed a significant decrease in weight at 18 months in the intervention group [17]. Moreover, Fernandez has presented the results of a meta-analysis that includes 20 studies which demonstrated that interventions to modify lifestyles improve anthropometric and clinical parameters at 3 months. At 6 and 12 months, the difference between the control and intervention groups is maintained, although with less precision [18].

Weight reduction and the practice of regular PA are possible in patients with psychosis and they improve their perception of health [3]. However, more research is needed with larger samples of subjects.

Interdisciplinary work is critical to approaching these disorders and the interventions. The joint work of the Primary Care Team [PCT] and Mental Health Team [MHT] is essential to developing physical care activities and health promotion as well as improving the care process of patients with psychosis. The early detection of CVRF and early symptoms, prioritized referral to primary care and promoting compliance and adherence are also relevant [19].

For this reason, the recommendations in the literature contemplate improving monitoring of CVRF in these patients and establishing channels of close collaboration between psychiatrists and the PCT as aims [20, 21].

Our group initiated a collaborative study between seven PCT and three reference MHT outpatient centers from which a joint protocol for monitoring CVRF emerged [22].

The outcome of this interest in improving the physical health of patients with psychosis is the present study. Its main objective is to evaluate the effectiveness of an intervention based on a program of PA and diet. It is a program coordinated between PCT and $\mathrm{MHT}$ to change the level of weekly PA, BMI and WC in patients with schizophrenia, schizoaffective or bipolar disorders. 
Secondary objectives will evaluate the effectiveness of this intervention to modify the cardiovascular risks, quality of life, tobacco consumption, dietary habits, blood pressure and plasma cholesterol and blood sugar levels.

\section{Methods/design}

\section{Study design}

Randomized clinical trial with a control group with one-year follow-up. Response variables will be assessed at 3, 6 and 12 months in comparison to baseline measurements (month 0 ) in the intervention group [IG] and control group [CG] (Figure 1).

\section{Setting}

Six public mental health centers and a residential center, all located in the metropolitan area of Bar- celona, participated. These centers serve patients referred by the corresponding PCT on an outpatient basis.

\section{Participants - Study Population}

Population residing to the geographical reference area of the MHT in Barcelona $(281,706)$ and Santa Coloma $(131,917)$, with the following selection criteria will be included:

\section{Inclusion criteria}

- Patients between 18 and 65 years of age with an antipsychotic drug treatment for at least 3 months prior to enrollment.

- Diagnosis recorded in the medical history: schizophrenia disorders (F20.* of ICD10), schizoaffective disorders (F25.* of ICD10) Bipolar disorder (F31.* of ICD10) and confirmed by their doctor through a clinical interview (DSM_IV criteria)

Figure 1: General flow of the design, number of patients and measurements taken in the follow-up

\section{Physical Activity}

- 4 weeks group PA in the health center

- 2 sessions of 1 hour/week

- 8 weeks PA walking outside of center

- 2 sessions of 1 hour/week

\section{Diet}

- 8 weeks

- 1 session of 40 minutes/week

\section{Intervention Group} $n=239$

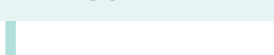

\section{Control Group} $n=239$

\section{Initial Evaluation}

3 mth. Evaluation

6 mth. Evaluation

$30 \%$ follow-up

losses
$30 \%$ follow-up losses 
- Residing in the reference area (area of influence of the MHT and PCT participants) for a minimum of one year.

- Knowledge of the Spanish language.

- Low level of PA (short version of the questionnaire, International Physical Activity Questionnaire, IPAQ). It means patients who do not perform moderate / high PA: 3 or more days of vigorous activity such as construction work, aerobics, cycling fast pace (requires hard physical effort and makes you breathe much harder than normal); or 5 or more days of moderate activity like carrying low weight, cycling at a steady pace (moderate physical effort and makes you breathe harder than normal). [23]

- BMI values equal or greater than 25 , so it includes patients with overweight $(25-29,9)$ and obesity (>30) [10].

\section{Exclusion criteria}

- Patients that present any contraindication (physical illness) for PA [24]

- Pregnancy or breastfeeding

- Having suffered an episode of acute mania or psychotic state one month before enrollment.

- Decompensation of the psychotic pathology from one month prior up to the time of recruitment measured by means of the Clinical Global Impression Scale [25]

- Dependence for substance abuse according to DSM-IV criteria with active consumption, except for nicotine dependence.

- Not visited by the MHT or by the PCT in the year prior to inclusion in the study

\section{Recruitment}

Selection will be made consecutively. The professionals from the MHT will recruit from among those visited in their centers. The recruitment period will be for about 6-12 months, taking advantage of the visits scheduled with the MHT or visits with the PCT.
The assessment of inclusion/exclusion will be made by the patient's psychiatrist.

\section{Sample size}

The sample size for each of the main measurements has been calculated and the one resulting in a larger sample size (BMI) has been chosen. Accepting an alpha risk of 0.05 and a beta risk of 0.20 in a bilateral contrast, it is necessary to study 478 subjects (239 subjects in the IG and 239 in the $\mathrm{CG}$ ) to detect a difference equal to or greater than $1.9 \mathrm{~kg} / \mathrm{m} 2$ in the BMI [26]. It is assumed that the common standard deviation is 6.2. A loss rate of $30 \%$ at follow-up has been estimated. It was decided to calculate the sample size using the BMI because we found quite a bit of literature in which this measure was used. However, we had no reference values in the IPAQ (the questionnaire used to assess physical activity) for SMD patients.

\section{Randomization}

After confirming that the criteria for inclusion has been met through the initial interview and once informed consent is signed, the distribution will be made at random by a computer program run by an outside researcher foreign to the professional recruiters to generate random numbers. They will be distributed so that in each center, half of the patients make up the IG patients and the other half make up the CG.

\section{Blinding}

Random assignment and collection of information will be carried out blind. During follow-up interviews the interviewer will advise the patient not to disclose whether the intervention has been done or not. Regardless, the percentage of patients who unmask the group to which they belong during the evaluation will be checked. 


\section{Materials Involved}

For measurements of physical variables, stadiometers, scales, tape measures, sleeves and blood pressure apparatus and other equipment necessary to perform the analysis will be used. The equipment is calibrated annually.

For the interventions, brochures with dietary and physical activity recommended, a food intake diary, notebooks, pens and pedometers will be handed out.

\section{Intervention design}

The subjects assigned to the CG will follow the usual practice with regular checks with their psychiatrist reference (usually every two months, if no decompensation) and the usual treatment of their disease.

Patients in both groups will be assigned the intervention protocol set that the team participating in the project elaborated [22]. Thus, patients with above normal parameters will be sent to their doctor to start a treatment plan.

The IG subjects will go through an educational program and carry out a PA program based on different stages as well as a dietary intervention in groups.

All the professional participants will receive training beforehand. The evaluators will undergo prior training by the research team (consisting of nurses, psychiatrists, family doctors and health technicians) about scales, physical measurements and the accurate collection of data.

Manuals on diet and PA have also been developed to facilitate the training of the professionals who will carry out the interventions.

\section{Physical Activity Intervention}

The frequency of the sessions will be 2 times a week with a duration of between 40 and 60 minutes each. The number of people per group will be a maximum of 15 people.

1. Familiarization phase (4 weeks, 40-minute sessions). Making contact with PA (intensity, recom- mendations for safe practices, etc...), pedometers and professionals bolster the program (MHT Nursing). The sessions will be held in the rooms of the participating centers or nearby public centers.

2. Phase of increasing the number of daily steps (8 weeks, sessions of 60 minutes). The aim is to increase the number of daily steps performed by each of the subjects to reach 10,000 steps a day (or as close as possible taking into account the number of initial steps). The MHT nurse who heads the group will guide and teach different routes adapted to the physical condition of the subjects located in the vicinity of the recruitment centers. During the last week, the routes will be delivered on paper with recommendations and strategies to continue to maintain the total number of steps.

\section{Dietary Intervention}

There will be 2 sessions per week of 20 minutes duration for 8 consecutive weeks to provide basic knowledge about healthy dietary habits.

They will be given by the nurses from the MHT or PCT centers in an entertaining and participatory manner. The nurses will record patient attendance in every session in order to assess the degree of compliance with the intervention.

The proposed diet is a traditional Mediterranean diet for cardiovascular protection with low levels of fat and cholesterol, high in fiber and unsaturated fats and a reduction in salt intake to $5 \mathrm{gr} /$ day.

At the end of each session, any doubts that may have arisen will be clarified and a diary of food eaten the last 24 hours will be looked over to review the knowledge acquired in each session.

Sessions 1 and 2. Content and objectives of the program and the importance of applying it to their daily lives. Proper diet.

Sessions 3 and 4. Welfare. Ideal Weight and BMI

Sessions 5 and 6. Calculation of BMI. Obesity and diseases.

Sessions 7 and 8. Care with overweight condition. Food pyramids. 
Sessions 9 and 10. Food Pyramid and portions. Nutritional requirements.

Sessions 11 and 12. Dietary Guidelines. Liquids.

Sessions 13 and 14. The making of a Mediterranean diet. Eating Habits.

Sessions 15 and 16. Hygiene, preservation and preparation of food.

\section{Outcomes}

\section{Primary outcomes}

- Level of physical activity: the International Physical Activity questionnaire (IPAQ), adapted to Spanish, will be used [27]. It allows for the assessment of PA as a continuous variable by calculating metabolic equivalent units [METs] per week and as a categorical variable, classifying subjects according to their level of PA (low, moderate or high). The IPAQ short form asks about three specific types of activity undertaken in the following domains: leisure time PA, domestic and gardening (yard) activities, work-related PA and transportrelated PA. The specific types of activity that are assessed are walking, moderate-intensity activities and vigorous-intensity activities. The items in the short IPAQ form were structured to provide separate scores on each type of activity. Computation of the total score for the short form requires summation of the duration (in minutes) and frequency (days) of walking, moderate-intensity and vigorous-intensity activities. Domain specific estimates cannot be estimated. This questionnaire has shown good validity and reliability in the general population [23].

- Body mass index (BMI): It is calculated from the weight in kilos divided by the square of the height in meters $\left(\mathrm{kg} / \mathrm{m}^{2}\right)$. A scale and a wall stadiometer with the patient barefoot and upright will be used. From the quantitative variable, a categorical variable will be calculated, in accordance with the consensus of the Spanish Society for the Study of Obesity that separates normal weight $(18,5-24,9)$, overweight $(25-29,9)$ and obesity (>30) [10].
- Waist circumference: The measurement is made with a flexible tape parallel to the ground, uncompressed, at the midpoint between the last rib and the iliac crest. The reading is taken at the end of a normal exhalation. The observer is placed on one side of the person to be measured. The upper limits of $102 \mathrm{~cm}$ for men and $88 \mathrm{~cm}$ for women are accepted as normal [28].

\section{Secondary outcomes}

- Cardiovascular Risk: Calculation using REGICOR tables to determine the risk of a coronary event in 10 years. It takes into account sex and age as non-modifiable risk factors and, in addition, blood pressure and cholesterol, smoking status and the presence or not of diabetes [29].

- Quality of Life: With the SF36 questionnaire, an instrument developed from an extensive battery of questionnaires used in the Medical Outcomes Study [30]. It detects states of positive and negative health and explores physical and mental health. It consists of 36 items that explore eight dimensions of the health status. In validation studies [31], The Cronbach alpha coefficient was higher than 0.7 in all dimensions, and the intraclass correlation coefficient stood between 0.58 to 0.99 .

- Tobacco: Smoker (number of cigarettes/day)/ Ex-smoker (not smoked in the last year)/smoker up to less than 12 months ago/Non-Smoker.

- HDL cholesterol, LDL cholesterol, total cholesterol, triglycerides, blood sugar: Units expressed in mg/100 ml. Collection method: venous puncture. A qualitative variable is calculated, with cutoffs of normality according to the ICS clinical practice guidelines, from each quantitative variable [32]. The initial analysis and follow-up will not be taken into account if there are any recorded in the last month. No analysis will be performed at six months.

- Blood pressure [BP]: Units expressed in $\mathrm{mmHg}$. Measurement method according to the measurement protocol. A qualitative variable is calculated good control/poor control [28]. 
- Dietary Habits: Using the 14-item Mediterranean diet fulfillment questionnaire used in the PREDIMED study [33]. The score ranges from 0 to 14 points, from minimum to maximum compliance. Consider 9 as the cutoff point [34].

\section{Confounding variable}

- Antipsychotic treatment prescribed to the patient: Active principle, dose, duration, therapeutic group. History of antipsychotic treatment: therapeutic groups and duration of each drug.

- Chronic disease: Ischemic heart disease: 121125; chronic obstructive pulmonary disease: J40-J44; Dyslipidemia: E78.0-E78.9; Hypertension: I10, I15; Diabetes mellitus: E10-E14; Asthma J45. Other relevant disease. The year of diagnosis will be noted. This information will be obtained through medical records and the clinical interview.

- Non-psychiatric active chronic medication: Recording of the active drugs (prescribed for at least 6 months) and their drug therapy group. The date of the prescription's start will be recorded.

- Visited by a PCT professional during the study period: number of visits

- Visited by a MHT professional during the study period: number of visits.

\section{Variables related to the patient's mental state}

- Brief Psychiatric Rating Scale, BPRS [35], developed to rapidly assess changes in the symptoms of patients with mental disorders. Actually, is one of the most frequently used instruments for evaluating psychopathology in patients with schizophrenia and in our setting is used as a measure of severity in these patients [36]. Validated in Spain in a version in the PANSS [37]. It consists of 18 items. It will be given to patients with schizophrenia or a schizoaffective disorder.

- Clinical Global Impression Scale (Clinical Global Impression, CGI) [38]. Applied scale that measures the severity of symptoms and improvement of symptoms due to therapeutic interventions. There is a version for schizophrenia in Spanish: it consists of two scales of five items each. Each item is scored using a Likert scale from 1 (normal) to 7 (severely ill). There is also a version for patients with bipolar disorder [39] and it is validated in Castilian [25].

- Universal Variables: Age (date of birth), Sex (male or female), employment status (employed, unemployed, retired, disability, pensioner or housewife), educational level (illiterate, literate, primary school, secondary school, university), property type (family home, protected home, residence), the center where the patient is recruited and where follow up is carried out during the study, the psychiatrist treating the patient (either public or private) and the GP and primary care team to which the patient is assigned.

\section{Data collection and follow-up (Table 1)}

The evaluation of the response variable will be done blind at 0, 3, 6 and 12 months. At three months, the intervention ends and its immediate effect will be evaluated. At 6 months, the midterm results will be evaluated. At 12 months, the long-term results will be evaluated.

Assessments will be conducted by evaluators (psychologists and mental health nurses) who do not participate in the intervention and are unaware of the group allocation of patients.

\section{Statistical analysis}

There will be an analysis of intention to treat. All patients who signed the informed consent will be included as well as those who have done the initial assessment.

Descriptive statistics will be calculated for the dependent, confounding and general variables for both the IG and the CG . The homogeneity of the two groups for these variables at baseline will also be checked.

In all cases, a bilateral alpha of 0.05 will be considered an error and confidence intervals will be calculated at 95\%. 
Table 1. measurements taken at each follow-up point.

\begin{tabular}{|c|c|c|c|c|}
\hline $\begin{array}{c}\text { Data } \\
\text { Collection }\end{array}$ & 0 mths. & 3 mths. & 6 mrhs. & 12 mths. \\
\hline \multicolumn{5}{|l|}{ Questionnaire } \\
\hline $\begin{array}{l}\text { socio- } \\
\text { demographic }\end{array}$ & $x$ & $x$ & $x$ & $x$ \\
\hline \multicolumn{5}{|c|}{ Physical Examination } \\
\hline Weight & $x$ & $x$ & $x$ & $x$ \\
\hline Height & $x$ & - & - & - \\
\hline $\begin{array}{l}\text { Waist } \\
\text { Circumference }\end{array}$ & $x$ & $x$ & $x$ & $x$ \\
\hline Blood Pressure & $x$ & $x$ & $x$ & $x$ \\
\hline \multicolumn{5}{|l|}{ Analysis } \\
\hline $\begin{array}{l}\text { Total } \\
\text { Colesterol, } \\
\text { HDL, LDL, } \\
\text { blood sugar y } \\
\text { triglycerides }\end{array}$ & $x$ & $x$ & - & $x$ \\
\hline \multicolumn{5}{|l|}{ Escalas } \\
\hline IPAQ & & $x$ & $x$ & $x$ \\
\hline $\begin{array}{l}\text { Mediterranean } \\
\text { Diet } \\
\text { Adherence }\end{array}$ & & $x$ & $x$ & $x$ \\
\hline BPRS & & $x$ & $x$ & $x$ \\
\hline CGI & & $x$ & $x$ & $x$ \\
\hline \multicolumn{5}{|c|}{ Clinical History Data } \\
\hline $\begin{array}{l}\text { Drug } \\
\text { Treatment }\end{array}$ & $x$ & $x$ & $x$ & $x$ \\
\hline $\begin{array}{l}\text { Chronic } \\
\text { Illnesses }\end{array}$ & $x$ & $x$ & $x$ & $x$ \\
\hline Visits to PCT & $x$ & $x$ & $x$ & $x$ \\
\hline Visits to MHC & $x$ & $x$ & $x$ & $x$ \\
\hline $\begin{array}{l}\text { Hospital } \\
\text { Admissions }\end{array}$ & $x$ & $x$ & $x$ & $x$ \\
\hline
\end{tabular}

An outcome variable will be calculated for each measurement point (3, 6 and 12 months) that is the difference between the result of $\mathrm{BMI}, \mathrm{WC}$ and weekly amount of PA at each cutoff and the initial value for each individual. The differences between groups will be evaluated and the 95\% confidence interval of the difference will be calculated.
An analysis of variance for repeated measures (ANOVA) to assess differences in the time of the dependent variables attributable to the effect of the intervention will be done. It will be adjusted for potential confounders: pharmacological treatment, care level of the intervention and the patient's mental state.

\section{Discussion}

The increased mortality in patients with severe mental illness is due in large part to the increased presence of cardiovascular risk factors. This study aims to demonstrate the effectiveness of a program of physical activity and diet coordinated between PCT and $\mathrm{MHT}$ to improve these factors. If the results are positive, their general level of implementation should be raised.

Throughout the implementation of the protocol, a number of limitations have been identified:

- Due to multicentrism of the study, centralized training of all participants in the study by the principal investigator is necessary.

- Due to the inherent characteristics of these patients, recruitment may be slower than expected.

- It is a clinical trial in which professionals know the group to which the patient belongs. In any case, an effort will be made to preserve masking in some study milestones such as randomization, the evaluation of the primary measurements and statistical analysis of the data.

- The loss that can be generated in the monitoring may be high, it being long-term and under normal conditions. An effort will be made to ensure patient adherence through telephone locating (at least three attempts per appointment, if the patient has stated that they want to participate) when miss any scheduled visits and intervention sessions.

- There may be a possible contamination effect between patients in the same center but of very low intensity, because, in principle, the patients have no relation between them. 
- It affects physical activity and diet but not smoking because we believe that is another type of addiction (to drugs ...).

- The generalizability of the study may be diminished by the exclusion of patients dependent on substances other than nicotine. We have taken this decision because patients are recruited in mental health centers. In our setting, patients with psychiatric illness but with a high dependence on drug use are referred to other types of centers called welfare centers for drug addicts. The latter centers are not involved in the study.

\section{Ethical aspects}

All patients enrolled will be informed verbally and in writing of the objectives, methodology, tests and interventions that they may receive if they participate in the study. Informed consent will be written in a language that the patient can understand. In case of disability, it must be signed by the legal guardian of the patient. Ethical aspects: the project has been evaluated and approved by the CEIC, the ethical committee of the Primary Healthcare-University Research Institute IDIAP Jordi Gol, with registration number P11/64.

\section{List of abreviations}

Severe Mental Disorder (SMD)

Cardiovascular Risk Factors (CVRF)

Metabolic Syndrome (MS)

Body Mass Index (BMI)

Waist Circumference (WC)

Physical Activity (PA)

Primary Care Team (PCT)

Mental Health Team (MHT)

Intervention Group (IG)

Control Group (CG)

Metabolic Equivalent Units (METs)

Blood Pressure (BP)

Brief Psychiatric Rating Scale (BPRS)

Clinical Global Impression (CGI)

\section{Author's contribution}

All authors participated in the conception and design of the study.

MIFS is coordinating the project and is responsible for the supervision of the assessors and professionals carrying out the intervention

RMF will monitor data collection and controls the quality of the same.

MIFS and RMF, together with a statistician will perform the data analysis.

MIFS and RMF will be responsible for the reporting and publication of results.

The entire team will participate in revising the article and in the final report.

\section{Author's information}

The research team and personnel involved in field work is made up of professionals at two levels of healthcare: primary care (family doctors and nurses in health centers) and mental health (psychiatrists and mental health nurses). In addition, the team has the assistance of physical education professionals, epidemiologists and statisticians.

\section{Acknowledgements}

Thanks to Carmen Martin Borras, who contributed to the development of the physical activity protocol, and to Eric L. Goode, the translator of this article.

\section{Competing interests}

The authors declare no conflicts of interests. A grant was obtained from the Institute of Health Carlos III for the project (PI11/01861) 


\section{References}

1. Perälä J, Suvisaari J, Saarni SI, Kuoppasalmi K, Isometsä E, Pirkola $S$, et al. Lifetime Prevalence of Psychotic and Bipolar I Disorders in a General Population. Arch Gen Psychiatry. 2007 Jan; 64(1): 19-28.

2. Bobes J, Arango C, Aranda P, Carmena R, Garcia-Garcia M, Rejas J; CLAMORS Study Collaborative Group. Cardiovascular and metabolic risk in outpatients with schizoaffective disorder treated with antipsychotics: results from the CLAMORS study. Eur Psychiatry. 2012 May; 27(4): 267-74.

3. Medeiros L, Obiols J, Navarro JB, Zúñiga A. Metabolic syndrome and health related quality of life in patients with schizophrenia. Actas Esp Psiquiatr 2013; 41(1): 17-26.

4. Laursen TM, Munk-Olsen T, Vestergaard M. Life expectancy and cardiovascular mortality in persons with schizophrenia. Curr Opin Psychiatry. 2012 Mar; 25(2): 83-8.

5. Bou Khalil R. Metabolic syndrome in patients with schizophrenia independently from atypical antipsychotics intake. Presse Med.2012May; 41(5): e238-43.

6. Bellivier F. Schizophrenia, antipsychotics and diabetes: Genetic aspects. Eur Psychiatry. 2005 Dec; 20 Suppl 4: S335-9.

7. Compton MT, Daumit GL, Druss BG. Cigarette smoking and overweight/obesity among individuals with serious mental illnesses: a preventive perspective. Harv Rev Psychiatry. 2006 Jul-Aug; 14(4): 212-22.

8. Aguilar E, Coronas R, Caixàs A. Síndrome metabólico en pacientes esquizofrénicos con tratamiento antipsicótico. Med Clin (Barc). 2012; 139(12): 542-546.

9. O'Donell CJ, Elosua R. Cardiovascular risk factors. Insights from Framingham Heart Study. Rev Esp Cardiol. 2006 Mar; 61(3): 299-310

10. Salas-Salvadó J, Rubio MA, Barbany M, Moreno B; Grupo Colaborativo de la SEEDO.Consenso SEEDO 2007 para la evaluación del sobrepeso y la obesidad y el establecimiento de criterios de intervención terapéutica. Med Clin (Barc). 2007 Feb; 128(5): 184-96.

11. Bernardo M, Grazia M. Esquizofrenia y Medicina de Familia. Implicaciones en Atención Primaria. Jano 2008; 1714: 37-44.

12. Tsoi DT, Porwal M, Webster AC. Interventions for smoking cessation and reduction in individuals with schizophrenia. Cochrane Database of Systematic Reviews 2010, Issue 6. Art. No.: CD007253

13. Faulkner G, Cohn T, Remington G. Interventions to reduce weight gain in schizophrenia. Cochrane Database of Systematic Reviews 2007, Issue 1. Art. No.: CD005148.

14. Holley J, Crone D, Tyson P, Lovell G. The effects of physical activity on psychological well-being for those with schizophrenia: $A$ systematic review. Br J Clin Psychol. 2011 Mar; 50(1): 84-105.

15. Bernard P, Ninot G. Benefits of exercise for people with schizophrenia: a systematic review. Encephale. 2012 Sep; 38(4): 280-7.
16. Wu MK, Wang CK, Bai YM, Huang CY, Lee SD. Outcomes of obese, clozapine-treated inpatients with schizophrenia placed on a six-month diet and physical activity program. Psychiatr Serv.2007 April; 58: 544-550.

17. Daumit G, Dickerson F, Wang N, Dalcin A, Jerome G, Anderson C, Young D, Frick K, Yu A, Gennusa J, Oefinger M, Crum R, Charleston J, Casagrande S, Guallar E, Goldberg R, Campbell L, Appel L. A Behavioral Weight-Loss Intervention in Persons with Serious Mental Illness. N Engl J Med. 2013 Mar 21.

18. Fernández-San-Martín MI, Martín-López LM, Masa-Font R, Olona-Tabueña N, Roman $Y$, Martin-Royo J, et al. The Effectiveness of Lifestyle Interventions to Reduce Cardiovascular Risk in Patients with Severe Mental Disorders: Meta-Analysis of Intervention Studies. Community Ment Health J. DOI 10.1007/ s10597-013-9614-6

19. Viron $M$, Baggett $T$, Hill $M$, Freudenreich $O$. Schizophrenia for primary care providers: how to contribute to the care of a vulnerable patient population. Am J Med. 2012 Mar; 125(3): 223-30.

20. Sáiz J, Bobes J, Vallejo J, Giner J, García-Portilla MP; Grupo de Trabajo sobre la Salud Física del Paciente con Esquizofrenia. Consensus on physical health of patients with schizophrenia from the Spanish societies of Psychiatry. Actas Esp Psiquiatr. 2008; 36: 251-64

21. Oud M, Meyboom-de Jong B. Somatic diseases in patients with schizophrenia in general practice: their prevalence and health care. BMC Family Practice 2009, 10: 32

22. Viñas L, Fernández M, San Emeterio L, Martín-López LM; Grup Psicosi Litoral. Detección de factores de riesgo cardiovascular en pacientes con psicosis. Aten Primaria. 2011 Jul; 43(7): 386-7. doi: 10.1016/j.aprim.2010.04.021.

23. Craig $C L$, Marshall $A L$, Sjöström $M$, Bauman $A E$, Booth $M L$, Ainsworth BE, et al. International Physical Activity Questionnaire: 12 country reliability and validity. Med Sci Sports Exerc. 2003; 35: 1381-95

24. Llibre blanc. Consens sobre les activitats preventives a l'edat adulta dins l'atenció primària. Barcelona (España): Direcció General de Salut Pública. Departament de Salud. Generalitat de Cataluña; 2005.

25. Vieta E, Torrent C, Martínez-Arán A, Colom F, Reinares M, Benabarre $A$ et al. Escala sencilla de evaluación del curso del trastorno bipolar: CGI-BP-M. Actas Esp Psiquiatr. 2002; 30: 301304.

26. Poulin MJ, Chaput JP, Simard V, Vincent P, Bernier J, Gauthier $Y$ et al. Management of antipsychotic-induced weight gain: prospective naturalistic study of the effectiveness of a supervised exercise programme. Australian and New Zealand Journal of Psychiatry. 2007; 41: 980-989.

27. Craig $C L$, Marshall $A L$, Sjostrom $M$, Bauman $A E$, Booth $M L$, Ainsworth $\mathrm{BE}$, et al. International physical activity questionnaire: 12-country reliability and validity. Med Sci Sports Exerc 2003; 35: $1381-95$ 
28. Marques A, Brotons C, Villar F, Lobos-Bejarano JM, Torcal J, Orozco-Beltrán, et al. Recomendaciones preventivas cardiovasculares PAPPS del 2009. Barcelona: semFYC ediciones, 2009: 101-113.

29. Marrugat J, Subirana I, Comin E, Cabezas C, Vila J, Elosua R, et al. Validity of an adaptation of the Framingham cardiovascular risk function: the VERIFICA study. J Epidemiol Community Health. 2007; 61: 40-7.

30. Ware JE. SF36 health survey update. Spine (Phila Pa 1976). 2000; 25: $3130-9$

31. Vilagut G, Ferrer M, Rajmil L, Rebollo P, Permanyer-Miralda G, Quintana JM, et al. The Spanish version of the Short Form 36 Health Survey: a decade of experience and new developments. Gac Sanit. 2005; 19(2): 135-50

32. Baena Diez JM, Barceló Colomer E, Ciurana Misol R, Franzi Sisó A, García Cordón MR, Rios Rodríguez MA, et al. Colesterol i risc coronari [guía en internet]. Barcelona: Institut Català de la Salut, 2009. Guies de pràctica clínica i material docent, núm 1 Disponible en: www.gencat.cat/ics/professionals/guies/ colesterol/colesterol.htm.

33. Martinez-Gonzalez MA, Fernandez-Jarne E, Serrano-Martinez M, Wright M, Gomez- Gracia E. Development of a short dietary intake questionnaire for the quantitative estimation of adherence to a cardioprotective Mediterranean diet. Eur J Clin Nutr. 2004; 58: 1550-2.

34. Estruch R, et al. Primary Prevention of Cardiovascular Disease with a Mediterranean Diet. N Engl J Med. 2013 Apr 4; 368(14): 1279-90

35. Overall JE, Gorham DR. The Brief Psychiatric Rating Scale. Pychological Reports. 1962; 10: 799-812

36. Casas E, Escandell MJ, Ribas M, Ochoa S. Instrumentos de evaluación en rehabilitación psicosocial. Rev. Asoc. Esp. Neuropsiq. 2010 Gen-Mar; 105: 25-47

37. Peralta V, Cuesta MJ. Validación de la escala de los síndromes positivo y negativo (PANSS) en una muestra de esquizofrénicos españoles. Actas Luso -Esp Neurol Psiquiatr. 1994; 4: 44-50.

38. Guy W. Clinical global impression (CGI). In: Guy W, editor. ECDEU Assessment Manual for Psychopharmacology. Rockville: US Department of Health, Education and Welfare, Public Health Service, Alcohol, Drug Abuse and Mental Health Administration, NIMH. Psychopharmacology Research Branch, Division of Extramural Research Programs; 1976. p. 218-22.

39. Spearing MK, Post RM, Leverich GS, Brandt D, Nolen W. Modification of the Clinical Global Impression (CGI) Scale for use in bipolar illness: the CGI-BP. Psychiatry Res. 1997; 73(3): 159-71.

\section{Comment on this article:}
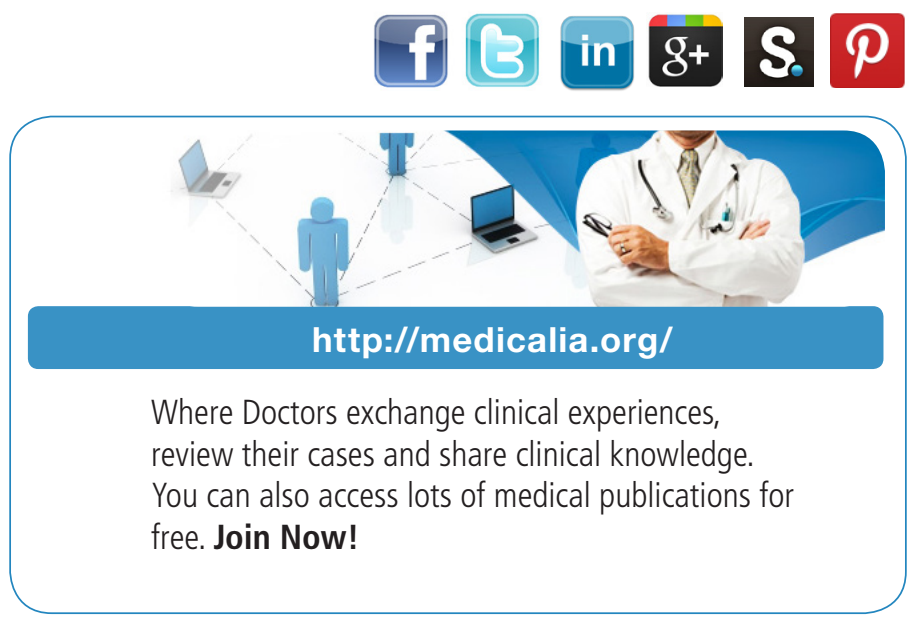

\section{Publish with iMedPub}

\section{http://www.imed.pub}

International Archives of Medicine is an open access journal publishing articles encompassing all aspects of medical science and clinical practice. IAM is considered a megajournal with independent sections on all areas of medicine. IAM is a really international journal with authors and board members from all around the world. The journal is widely indexed and classified Q1 in category Medicine. 